How to cite this paper:

Tran, Q. T., Li, H., \& Trinh, Q. K. (2019). Cellular network traffic prediction using exponential smoothing Methods. Journal of Information and Communication Technology, $18(1), 1-18$.

\title{
CELLULAR NETWORK TRAFFIC PREDICTION USING EXPONENTIAL SMOOTHING METHODS
}

\author{
${ }^{1,2}$ Quang Thanh Tran, ${ }^{1}$ Li Hao \& ${ }^{2}$ Quang Khai Trinh \\ ${ }^{1}$ Key Lab of Information Coding and Transmission, Southwest Jiaotong \\ University, China \\ ${ }^{2}$ Faculty of Electrical-Electronic Engineering, University of Transport \\ and Communications, Vietnam
}

thanhtq@utc.edu.vn; lhao@swjtu.edu.cn; khaitq@utc.edu.vn

\begin{abstract}
Wireless traffic prediction plays an important role in network planning and management, especially for real-time decision making and short-term prediction. Systems require high accuracy, low cost, and low computational complexity prediction methods. Although exponential smoothing is an effective method, there is a lack of use with cellular networks and research on data traffic. The accuracy and suitability of this method need to be evaluated using several types of traffic. Thus, this study introduces the application of exponential smoothing as a method of adaptive forecasting of cellular network traffic for cases of voice (in Erlang) and data (in megabytes or gigabytes). Simple and Error, Trend, Seasonal (ETS) methods are used for exponential smoothing. By investigating the effect of their smoothing factors in describing cellular network traffic, the accuracy of forecast using each method is evaluated. This research comprises a comprehensive analysis approach using multiple case study comparisons to determine the best fit model. Different exponential smoothing models are evaluated for various traffic types in different time scales. The experiments are implemented on real data from a commercial cellular network, which is divided into a training data part for modeling and test data part for forecasting comparison.
\end{abstract}


This study found that ETS framework is not suitable for hourly voice traffic, but it provides nearly the same results with HoltWinter's multiplicative seasonal (HWMS) in both cases of daily voice and data traffic. HWMS is presumably encompassed by ETC framework and shows good results in all cases of traffic. Therefore, HWMS is recommended for cellular network traffic prediction due to its simplicity and high accuracy.

Keywords: Cellular network traffic, exponential smoothing, Holt-Winter's multiplicative seasonal, wireless traffic prediction.

\section{INTRODUCTION}

Wireless traffic prediction is a key component of network planning, development, and management. Accurate prediction will become even more necessary with the development of $5^{\text {th }}$ generation wireless systems $(5 \mathrm{G})$ that contain many new service capabilities (5G PPP, 2015). The 5G system has a higher capacity and higher density of mobile broadband users than the current $4 \mathrm{G}$ system. It also supports device-to-device communications and massive machine-type communications (NGMN Alliance, 2015). Consequently, people are living in the age of social networks (Tyagi \& Kumar, 2017) and the Internet-of-Things (Matta, Pant, \& Arora, 2017). Life becomes more convenient and intelligent when everything can be connected via heterogeneous wireless networks (Qiang, Li, \& Altman, 2017). Along with these advanced technologies, YusufAsaju, Dahalin, and Ta'a (2018) also figured out the issues of mobile network performance and proposed a framework for modeling mobile network quality of experience using the big data analytics approach. And in fact, better network operation and management are required to ensure a robust infrastructure that includes the underlying network and supporting technologies, for example.

Analysis of wireless network traffic shows that the traffic series normally contains seasonal components and can be modeled and forecasted by time series analysis models (Tran, Ma, Li, Hao, \& Trinh, 2015). Authors in these papers proposed combining statistical procedures for modeling and forecasting cellular network traffic, such as the autoregressive integrated moving average (ARIMA) and generalized autoregressive conditional heteroskedasticity (GARCH). They took advantage of the ARIMA model for capturing the conditional mean of the traffic series and the GARCH model for dealing with the conditional heteroskedasticity existing inside the traffic. They achieved better forecast results compared with the individual models, but at the cost of computational complexity. The results can be used for capacity planning and overload warning issues that are important parts of network planning.

Exponential smoothing is a simple method of adaptive forecasting in which the forecasts adjust based on past errors, unlike forecasts from regression models that use fixed coefficients. Exponential smoothing 
methods have been applied in several areas, such as palm oil real production forecasting (Siregar, Butar-Butar, Rahmat, Andayani, \& Fahmi, 2017), power (Usaratniwart, Sirisukprasert, Hatti, \& Hagiwara, 2017), revenue forecasting (Rahman, Salma, Hossain, \& Khan, 2016), and solar irradiance prediction (Margaret \& Jose, 2015), to name a few. These researchers all achieved good results with this low-complexity and low-cost method. In terms of wireless traffic prediction, Tikunov and Nishimura (2007) proposed the application of Holt-Winter's exponential smoothing, which is simple, low cost, does not require a without highly skilled analyst, and operates nearly automatically for GSM/GPRS network Erlang traffic prediction. The recorded data were classified into three types, namely high, medium, and low intensity traffic cells. The authors focused on cells with high and medium traffic intensity for the purposes of overload warning and capacity planning. Although good results were achieved, only voice traffic was considered. In the era of data, there is a necessity for more comprehensive studies about using exponential smoothing in cellular network traffic that includes not only voice (Erlang) but also data (megabytes or gigabytes).

Base on the mentioned requirement, more exponential smoothing methods were investigated that included not only the specific Holt-Winter's multiplicative seasonal method (HWMS), but also different types of exponential smoothing methods. They were then applied to forecast cellular network traffic that consists of not only voice (in Erlang) but also data (in megabytes or gigabytes). In this study, the simple exponential smoothing methods include single, double, Holt-Winter's no seasonal, Holt-Winter's additive seasonal, and HWMS. The methods are introduced together with an Error, Trend, Seasonal (ETS) framework. The exponential smoothing methods are considered in three cases of hourly voice, daily voice, and daily data traffic types.

\section{SIMPLE EXPONENTIAL SMOOTHING}

Simple exponential smoothing methods include:

- Single smoothing: one parameter $0<\alpha \leq 1$,

- Double smoothing: one parameter $0<\alpha \leq 1$,

- Holt-Winters - No seasonal: two parameters $0<\alpha, \beta<1$,

- Holt-Winters - Additive seasonal: three parameters $0<\alpha, \beta, \gamma<1$, and

- Holt-Winters - Multiplicative seasonal: three parameters $0<\alpha, \beta, \gamma<1$

where $\alpha, \beta$, and $\gamma$ are the damping, or smoothing, factors. 
The analysis of cellular network traffic is appropriate for the HWMS method (Valakevicius \& Brazenas, 2015), which is suitable for a series with a linear time trend and multiplicative seasonal variation. If $x_{t}$ is the input traffic series, then the smoothed series, $\mathrm{pa}^{\text {is given by }}$

$$
\hat{x}_{t+i}=(a+b i) c_{t+i}
$$

where $a$ is the permanent component (intercept), $b$ is the trend, and $c_{t}$ is the multiplicative seasonal factor. These three coefficients are defined by the following recursions:

$$
\begin{aligned}
& a(t)=\alpha \frac{x_{t}}{c_{t}(t-s)}+(1-\alpha)(a(t-1))+b(t-1) \\
& b(t)=\beta(a(t)-a(t-1))+(1-\beta) b(t-1) \\
& c_{t}(t)=\gamma \frac{x_{t}}{a(t)}+(1-\gamma) c_{t}(t-s)
\end{aligned}
$$

where $<\alpha, \beta$, and $\gamma<1$ are the damping factors and $s$ is the seasonal frequency.

The forecasts are computed by:

$$
\hat{x}_{t+i}=(a(T)+b(T) i) c_{T+i-s}
$$

where the seasonal factors are used from the last $s$ estimates.

\section{ERROR TREND SEASONAL EXPONENTIAL SMOOTHING}

This framework defines an extended class of exponential smoothing methods and offers a theoretical foundation for analysis of these models using state-space based likelihood calculations. Support for model selection and calculation of forecast standard errors are also included. The standard exponential smoothing models discussed in the previous section, such as HWMS, are encompassed by this ETS framework.

In the ETS exponential smoothing method, the time series may be decomposed into three components, namely the error (E) that is the irregular unpredictable component of the series, the trend $(\mathrm{T})$ that characterizes the long-term movement of the time series, and the season (S) that corresponds to a pattern with known periodicity. 
The ETS models can be described as state equations that are extended versions of those outlined by Hyndman et al. (2002) as in Equations (6), (7), and (8), respectively:

$$
\begin{aligned}
& l_{s}=\alpha P\left(x_{t-1}, e_{t}\right)+(1-\alpha) Q\left(x_{t-1}\right) \\
& b_{t}=\beta R\left(x_{t-1}, e_{t}\right)+(1-\beta) \phi_{1} b_{t-1}^{\phi_{2}} \\
& s_{t}=\gamma T\left(x_{t-1}, e_{t}\right)+(1-\gamma) s_{t-m}
\end{aligned}
$$

where $l$ is a level term, $b$ is a growth term, and $s$ is a seasonal term. Variables $P, R$, and $T$ are functions of the prediction error and lagged states; $Q$ is a function of the lagged state; $\phi_{1}$ is the damping parameter for linear trend models; $\phi_{2}$ is the damping parameter for multiplicative trend models; and $e_{t} \sim \operatorname{iid}\left(0, \sigma^{2}\right)$ is prediction error. Figure 1 presents the model of ETS exponential smoothing method.

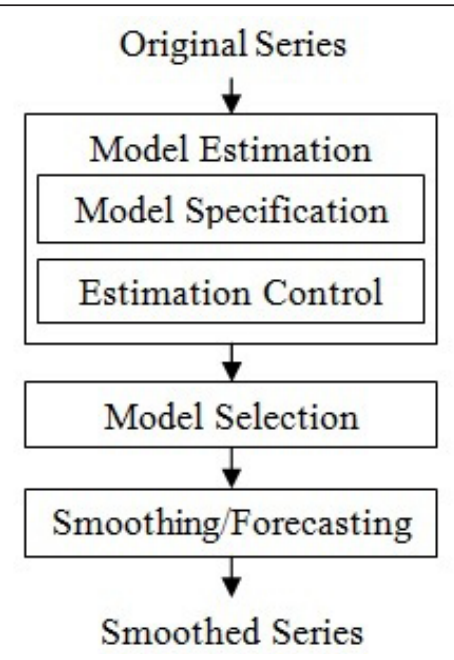

Figure 1. Model of ETS Exponential Smoothing Method.

\section{Model Estimation}

\section{Model Specification}

In this section, the type of ETS model used for smoothing is specified. There are a total of 30 possible ETS models based on the various combinations of the three components, E, T, and S, as defined in Equations (9), (10) and (11), respectively: 


$$
\begin{aligned}
& E\{A, M\} \\
& T\{N, A, M, A D, M D\} \\
& S\{N, A, M\}
\end{aligned}
$$

where $N=$ none, $A$ = additive, $M=$ multiplicative, $A D=$ additive dampened, and $M D=$ multiplicative dampened.

\section{Estimation Control}

According to the chosen ETS model specification, the corresponding unknown parameters $\theta=(\alpha, \beta, \gamma, \phi)$, and the initial states $x_{0}$ may be estimated using either the maximum likelihood or average mean square error minimization (AMSE) methods.

The Gaussian log likelihood for ETS specifications can be written in terms of the prediction errors, as in Equation (12):

$$
\log L\left(\theta, x_{0}, \sigma^{2} \mid y\right)=-\frac{2}{T} \log \left(2 \pi \sigma^{2}\right)-\frac{1}{2} \sum_{t-1}^{n} \frac{e_{t}^{2}}{\sigma^{2}} .
$$

The parameters and initial states are achieved by maximizing the likelihood in Equation (12) with respect to and using the Broyden, Fletcher, Goldfarb, and Shanno (BFGS) algorithm (Fletcher, 1987).

The average mean square error (AMSE) of the h-step forecasts is expressed as in Equation (13):

$$
A M S E=\frac{1}{T} \sum_{k=1}^{h}\left(\frac{1}{h} \sum_{k=1}^{h} e_{t+k \mid t}^{2}\right) .
$$

The parameters and initial states that minimize the AMSE using BFGS are then achieved.

\section{Model Selection}

In this step, the model can be selected based on either the comparison of a likelihood-based information criterion across the models to decide which one more closely fits the data or the forecast error, i.e., an out-of-sample AMSE to decide which one has a more accurate forecast result.

The likelihood-based information criteria include Akaike information criterion (AIC), Schwarz information criterion (BIC), or the Hannan-Quinn criterion (HQ), as expressed in Equations (14), (15), and (16), respectively: 


$$
\begin{array}{lcc}
A I C & = & -2 \log L\left(\hat{\theta}, \hat{x}_{0}\right)+2 p \\
B I C & = & -2 \log L\left(\hat{\theta}, \hat{x}_{0}\right)+\log (T) p \\
H Q & = & -2 \log L\left(\hat{\theta}, \hat{x}_{0}\right)+2 \log (\log T) p
\end{array}
$$

where $\left(\hat{\theta}, \hat{x}_{0}\right)$ are the maximized values and $p$ is the number of parameters in $\hat{\theta}$ plus the number of the estimated initial states in $\hat{x}_{0}$. The chosen model is the one with the minimum value of AIC (BIC, or HQ).

The out-of-sample AMSE is given in Equation (17):

$$
A M S E=\frac{1}{T^{*}} \sum_{t=T+1}^{T+T^{*}}\left(\frac{1}{h} \sum_{k=1}^{h} e_{t+k \mid t}^{2}\right) .
$$

\section{Smoothing/Forecasting}

Using the chosen ETS model with estimated parameters, the in-sample smoothed series can be achieved by the one-step-ahead forecast function, $h\left(x_{t-1}, \theta\right)$. Based on the smoothing model and the estimated parameters, as well as the in-sample data, the out-of-sample forecasted values can be obtained by implementing the dynamic forecast of the series.

\section{EXPERIMENTAL METHODOLOGY AND RESULT}

The real traffic from commercial cellular networks was collected and included voice in Erlang and data in Mbps. The first trace is 31-day hourly voice traffic collected during the New Year period from MSCID of MSC Hanoi1. The traffic data ranged from 00:00 December 9th, 2015 until 23:00 January 8th, 2016 and included 744 observations. The second trace is the daily voice traffic and included $2363 \mathrm{G}$ traffic values that ranged from March 9th until October 30th, 2014, collected from 110 siteBs in the CauGiay district area in Hanoi. The third trace is the $3 \mathrm{G}$ data collected from 600 cells located within the HoanKiem district in Hanoi. The 443-value daily data traffic series ranged from September 12th, 2014 to November 28th, 2015.

Exponential smoothing methods were applied to forecast these three traces that are distinguished from each other by traffic type, namely voice vs. data and hourly vs. daily. The simple exponential smoothing methods included single, double, and Holt-Winters and were used to implement the forecasts for the three traffic traces. Then, the achieved models that gave the 
best results were chosen to make comparisons with the best choices by the ETS exponential smoothing framework in terms of estimation information, in-sample forecast, and out-of-sample forecast.

\section{Hourly Voice Traffic}

The simple exponential smoothing methods were applied to hourly voice traffic. The estimation outputs are shown in Table 1, where HWMS presents the best results in terms of the 426.5057 RMSE. Thus, the HWMS was chosen as the best forecasting method in the case of hourly voice traffic. In contrast, the ETS framework was also applied to this hourly voice traffic, and the estimation outputs are listed in Table 2 . The numerical results indicate that this framework is not suitable for the hourly data series when both AIC-based and AMSE-based models yield abnormal RMSE values. To confirm this judgment, we further implement the in-sample and out-of-sample forecast tests for these two models and compare the results with those obtained using HWMS. The results are shown in Figures 2 and 3 which display that the HWMS out-ofsample forecasted data is very close to the original data, so we can figure out that HWMS outperforms the two ETS models in modeling and forecasting hourly cellular voice traffic.

Table 1

Hourly Voice Traffic Estimation Outputs of Simple Exponential Smoothing Methods.

\begin{tabular}{|c|c|c|c|c|c|c|}
\hline \multicolumn{2}{|c|}{ Fxnonentialsmoothing Methods } & Single & Double & HWNS & HWAS & HWMS \\
\hline \multirow{3}{*}{ Parameters } & Alpha & 0.9990 & 0.9990 & 1.0000 & 1.0000 & 1.0000 \\
\hline & Beta & & & 1.0000 & 0.0000 & 0.0000 \\
\hline & Gamma & & & & 0.0000 & 0.0000 \\
\hline \multicolumn{2}{|c|}{ Sum of Squared Residuals } & $5.36 \mathrm{E}+09$ & $4.15 \mathrm{E}+09$ & $4.05 E+09$ & $2.36 \mathrm{E}+08$ & $1.35 \mathrm{E}+08$ \\
\hline \multicolumn{2}{|c|}{ Root Mean Squared Error } & 2684.505 & 2363.002 & 2334.268 & 563.0144 & 426.5057 \\
\hline \multirow{2}{*}{$\begin{array}{l}\text { End of Period } \\
\text { Levels }\end{array}$} & Mean & 2797.097 & 2793.238 & 2793.240 & 9059.919 & 10147.16 \\
\hline & Trend & & -3854.95 & -3850.91 & 1.419014 & 1.419014 \\
\hline
\end{tabular}


Table 2

Hourly Voice Traffic Estimation Outputs of AIC and AMSE Based ETS Chosen Models.

\begin{tabular}{lll}
\hline & AIC Based & AMSE Based \\
Chosen Models & & \\
\hline $\mathrm{E}$ & $\mathrm{E}\{\mathrm{M}\}$ & $\mathrm{E}\{\mathrm{A}\}$ \\
$\mathrm{T}$ & $\mathrm{T}\{\mathrm{MD}\}$ & $\mathrm{T}\{\mathrm{MD}\}$ \\
$\mathrm{S}$ & $\mathrm{S}\{\mathrm{N}\}$ & $\mathrm{S}\{\mathrm{M}\}$ \\
Parameters & & \\
Alpha & 1.000000 & \\
Beta & 0.986463 & 1.000000 \\
Gamma & & 0.000000 \\
Phi & 1.000000 & 0.000000 \\
Estimation information & & 1.000000 \\
AIC & 16065.87 & \\
SSR & 95.84155 & 16665.64 \\
RMSE & 0.358914 & $5.25 \mathrm{E}+09$ \\
AMSE & $3.21 \mathrm{E}+40$ & 2656.054 \\
& & 21744630
\end{tabular}

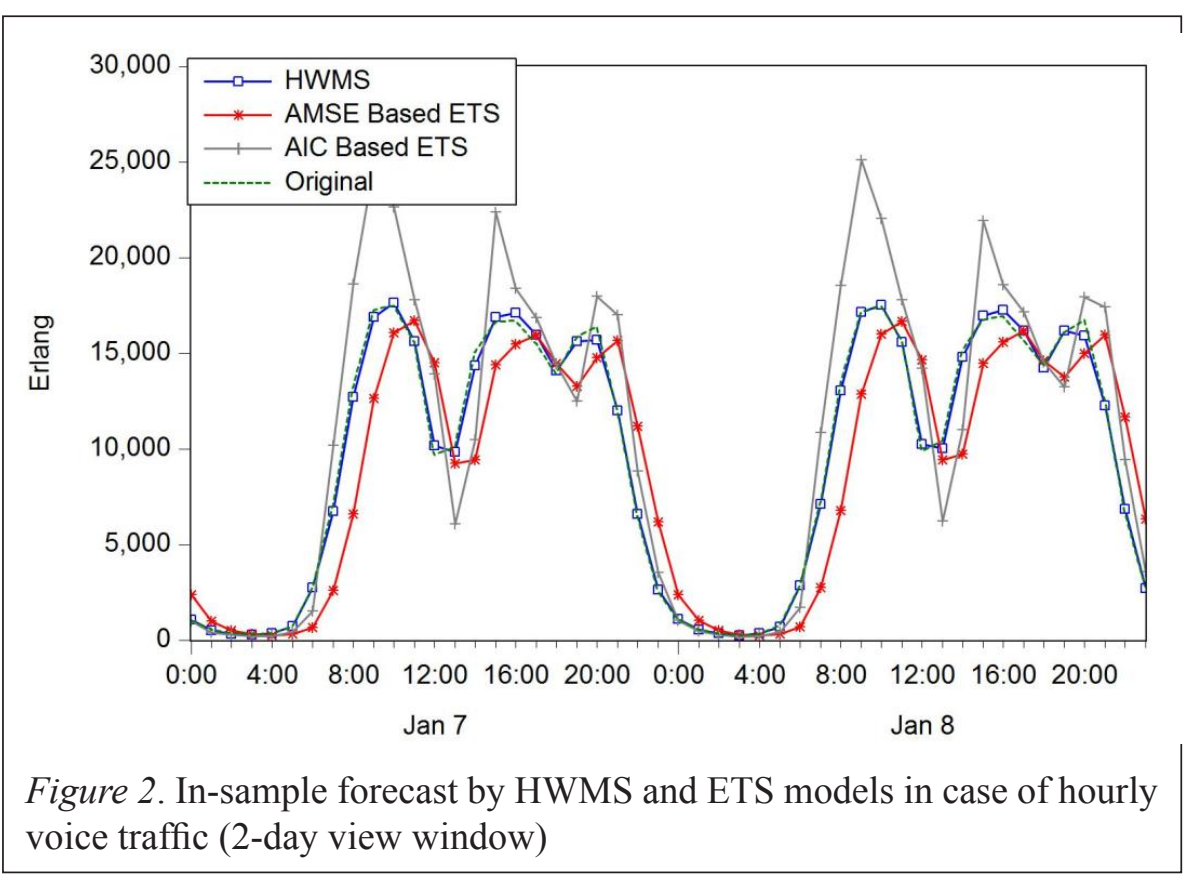




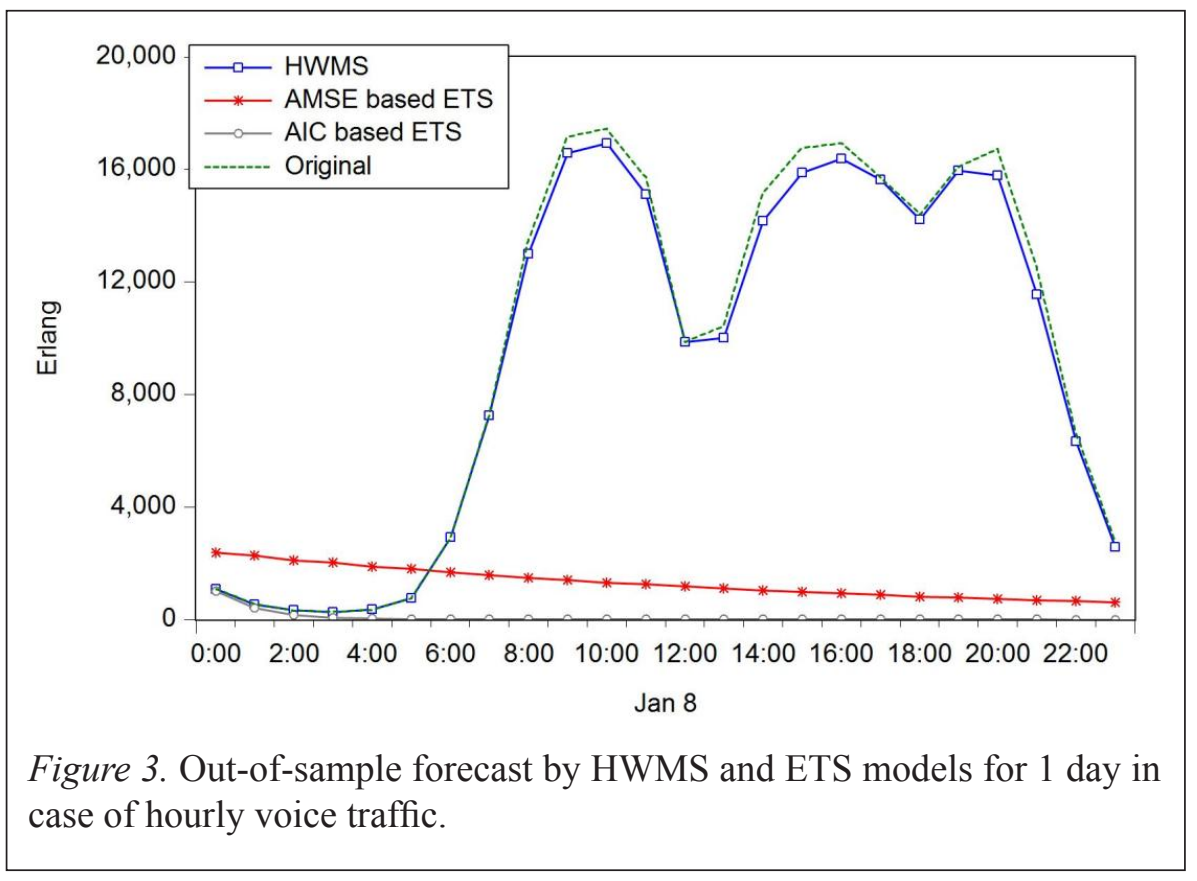

\section{Daily Voice Traffic}

The same experimental procedure was implemented for the case of daily voice traffic. Table 3 illustrates the estimation outputs of the simple exponential smoothing methods. The HWMS again presents the best results among other methods in term of RMSE, which is 531.6792. Thus, HWMS was chosen as the best forecasting method for the case of daily voice traffic. The ETS framework was also applied to the daily voice traffic, and the estimation outputs are illustrated in Table 4. Based on the results, the AIC-based ETS model, i.e. $\{\mathrm{A}, \mathrm{N}, \mathrm{M}\}$, should be chosen due to the better RMSE.

Table 3

Daily Voice Traffic Estimation Outputs of Simple Exponential Smoothing Methods.

\begin{tabular}{|c|c|c|c|c|c|c|}
\hline \multicolumn{2}{|c|}{ Exponential Smoothing Methods } & \multirow{2}{*}{$\frac{\text { Single }}{0.0010}$} & \multirow{2}{*}{$\begin{array}{c}\text { Double } \\
0.0070\end{array}$} & \multirow{2}{*}{$\frac{\text { HWNS }}{0.1100}$} & \multirow{2}{*}{$\begin{array}{c}\text { HWAS } \\
0.8200\end{array}$} & \multirow{2}{*}{$\frac{\text { HWMS }}{0.8400}$} \\
\hline \multirow{3}{*}{ Parameters } & Alpha & & & & & \\
\hline & Beta & & & 0.0000 & 0.0000 & 0.0000 \\
\hline & Gamma & & & & 0.0000 & 0.0000 \\
\hline \multicolumn{2}{|c|}{ Sum of Squared Residuals } & $3.84 \mathrm{E}+08$ & $3.89 \mathrm{E}+08$ & $4.33 \mathrm{E}+08$ & 73565891 & 66713129 \\
\hline
\end{tabular}

(continued) 


\begin{tabular}{|c|c|c|c|c|c|c|}
\hline \multicolumn{2}{|c|}{ Exponential Smoothing Methods } & Single & Double & HWNS & HWAS & HWMS \\
\hline \multicolumn{2}{|c|}{ Root Mean Squared Error } & 1275.546 & 1283.683 & 1354.245 & 558.3188 & 531.6792 \\
\hline \multirow{2}{*}{$\begin{array}{l}\text { End of Period Levels } \\
\text { Levels }\end{array}$} & Mean & 5259.193 & 5440.615 & 5707.966 & 5691.035 & 5662.484 \\
\hline & Trend & & 2.008049 & 9.387678 & 1.317833 & 1.317833 \\
\hline
\end{tabular}

Table 4

Daily Voice Traffic Estimation Output of AIC and AMSE Based ETS Chosen Models.

\begin{tabular}{lll}
\hline & AIC Based & AMSE Based \\
Chosen Models & & \\
\hline & & \\
$\mathrm{E}$ & $\mathrm{E}\{\mathrm{A}\}$ & $\mathrm{E}\{\mathrm{A}\}$ \\
$\mathrm{T}$ & $\mathrm{T}\{\mathrm{N}\}$ & $\mathrm{T}\{\mathrm{A}\}$ \\
$\mathrm{S}$ & $\mathrm{S}\{\mathrm{M}\}$ & $\mathrm{S}\{\mathrm{M}\}$ \\
Parameters & & \\
Alpha & & 0.000000 \\
Beta & 0.838877 & 0.000000 \\
Gamma & & 0.000000 \\
Phi & 0.000000 & \\
Estimation information & & \\
AIC & & 4363.242 \\
SSR & 4269.541 & 97473742 \\
RMSE & 66652447 & 642.6697 \\
AMSE & $\mathbf{5 3 1 . 4 3 7 3}$ & 412858.1 \\
& 484775.5 & \\
\hline
\end{tabular}

The comparison between the HWMS and ETS models was done next for the case of daily voice traffic. It can be seen from Table 5 that the achieved ETS model shows the same parameters as HWMS. In addition, there appears to be no difference between the HWMS and ETS $\{A, N, M\}$ in terms of in-sample forecasts in Figures 4 and 5, and the out-of-sample forecast in Figure 6. 
Table 5

Comparison of HWMS and ETS Methods in Case of Daily Voice Traffic.

\begin{tabular}{|c|c|c|c|}
\hline \multirow{2}{*}{\multicolumn{2}{|c|}{ Exponential Smoothing Methods }} & Simple & ETS \\
\hline & & HWMS & $\{\mathrm{A}, \mathrm{N}, \mathrm{M}\}$ \\
\hline \multirow{4}{*}{ Parameters } & Alpha & \multirow[t]{2}{*}{0.838877} & \multirow[t]{2}{*}{0.838877} \\
\hline & Beta & & \\
\hline & Gamma & \multirow[t]{2}{*}{0.000000} & \multirow[t]{2}{*}{0.000000} \\
\hline & Phi & & \\
\hline \multicolumn{2}{|c|}{ Akaike Information Criterion } & & 4269.541 \\
\hline \multicolumn{2}{|c|}{ Sum of Squared Residuals } & 66713129 & 66652447 \\
\hline \multicolumn{2}{|c|}{ Root Mean Squared Error } & 531.6792 & 531.4373 \\
\hline \multicolumn{2}{|c|}{ Average Mean Squared Error } & & 484775.5 \\
\hline
\end{tabular}

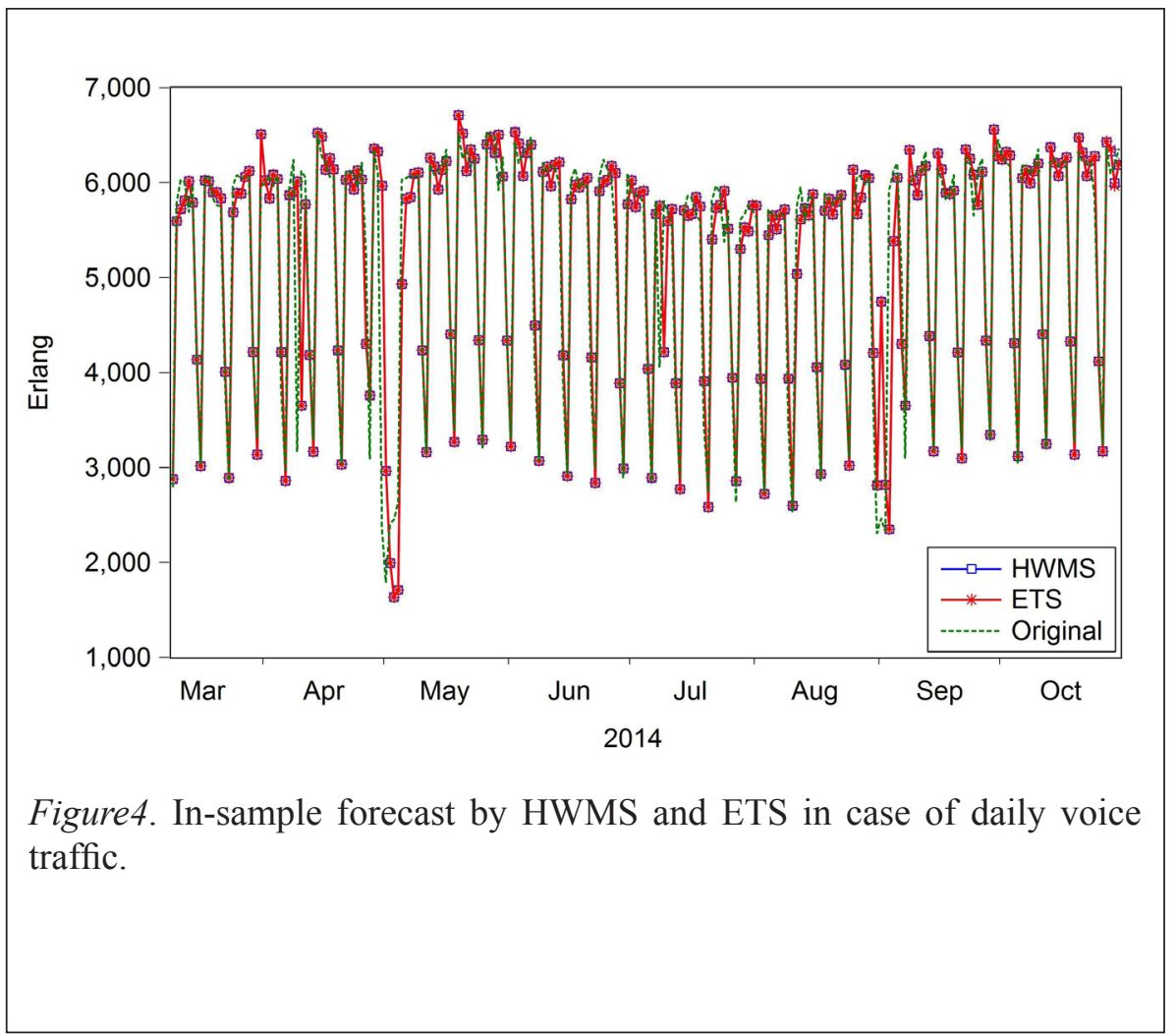




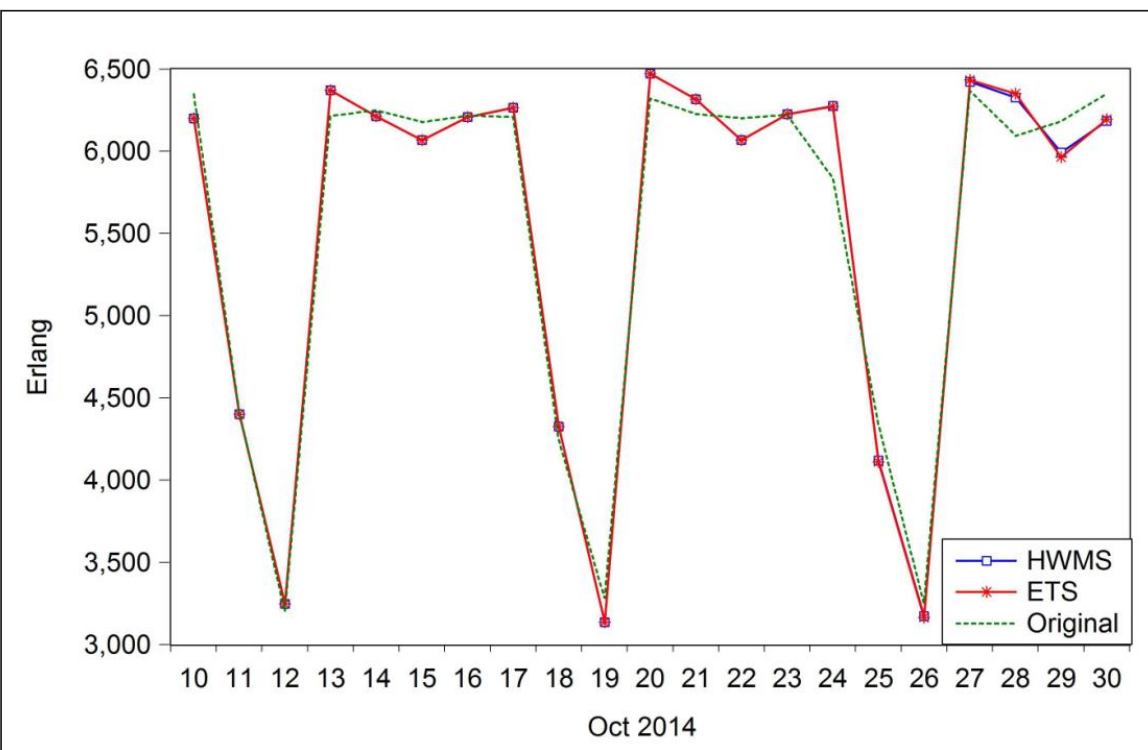

Figure 5. In-sample forecast by HWMS and ETS for 3 weeks in case of daily voice traffic.

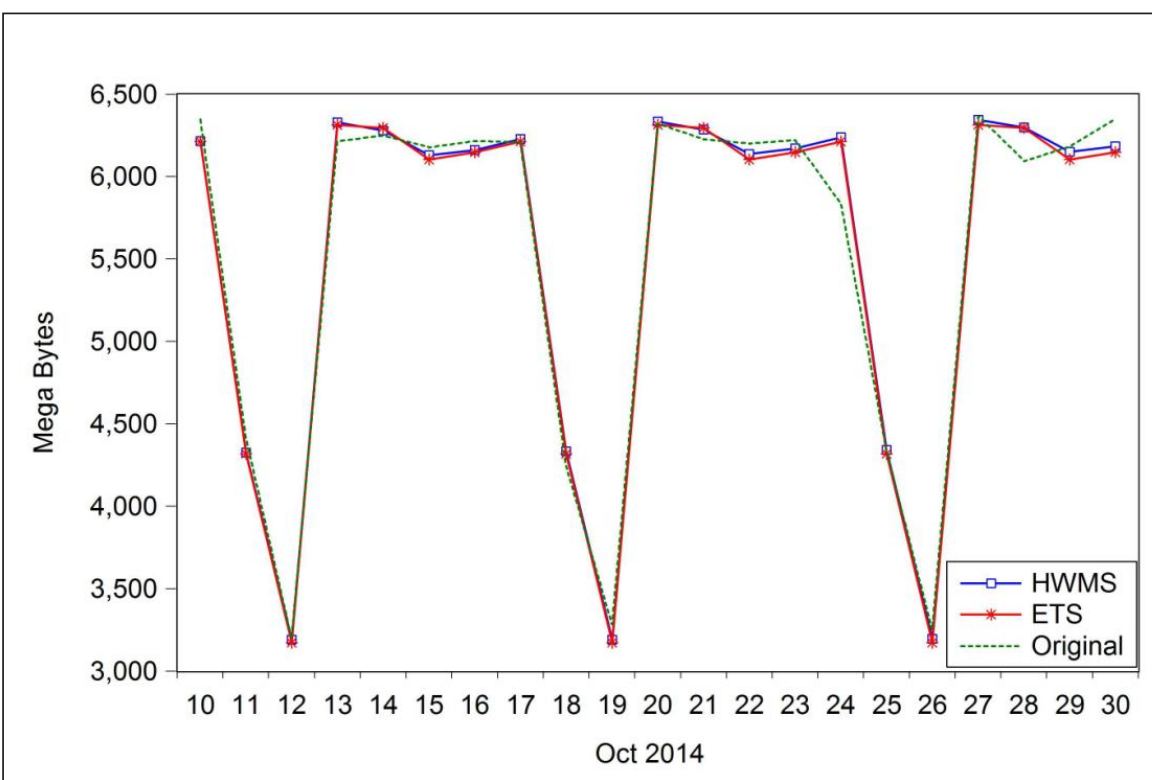

Figure 6. Out-of-sample forecast by HWMS and ETS for 3 weeks in case of daily voice traffic. 


\section{Daily Data Traffic}

The HWMS again is the best choice among other the simple exponential smoothing methods in the case of daily data traffic due to the best RMSE of 48.03080, as presented in Table 6. Besides, AIC-based ETS $\{$ A,N,M was also the chosen model with the lower RMSE of 48.03521, as shown in Table 7. The same results were achieved in the case of daily data traffic in which the HWMS and ETS $\{A, N, M\}$ have almost the same parameters, as in Table 8. The same is true for the same in-sample forecasts in Figures 7 and 8 and the same out-of-sample forecast in Figure 9.

Table 6

Daily Data Traffic Estimation Outputs of Simple Exponential Smoothing Methods.

\begin{tabular}{|c|c|c|c|c|c|c|}
\hline $\begin{array}{l}\text { Exponential } \\
\text { Methods }\end{array}$ & Smoothing & Single & Double & HWNS & HWAS & HWMS \\
\hline \multirow{3}{*}{ Parameters } & Alpha & 0.8080 & 0.1140 & 0.3200 & 0.6600 & 0.6700 \\
\hline & Beta & & & 0.0000 & 0.0000 & 0.0000 \\
\hline & Gamma & & & & 0.0000 & 0.0000 \\
\hline \multicolumn{2}{|c|}{ Sum of Squared Residuals } & 2943240 . & 3114186. & 2932171. & 1040425. & 1021982. \\
\hline \multicolumn{2}{|c|}{ Root Mean Squared Error } & 81.51002 & 83.84370 & 81.35660 & 48.46225 & 48.03080 \\
\hline \multirow{2}{*}{ End of Period Levels } & Mean & 1042.763 & 1117.194 & 1113.848 & 1093.138 & 1094.759 \\
\hline & Trend & & 1.345373 & 1.359729 & 0.878545 & 0.878545 \\
\hline
\end{tabular}

Table 7

Daily Data Traffic Estimation Output of AIC and AMSE Based ETS Chosen Models.

Chosen Models

AIC Based

AMSE Based

\begin{tabular}{lll}
\hline E & $\mathrm{E}\{\mathrm{A}\}$ & \\
$\mathrm{T}$ & $\mathrm{T}\{\mathrm{N}\}$ & $\mathrm{E}\{\mathrm{A}\}$ \\
$\mathrm{S}$ & $\mathrm{S}\{\mathrm{M}\}$ & $\mathrm{S}\{\mathrm{MD}\}$ \\
Parameters & & \\
Alpha & 0.669730 & 0.457427 \\
Beta & & 0.000000 \\
Gamma & 0.000000 & 0.000000 \\
Phi & & 0.729664 \\
\hline
\end{tabular}

(continued) 


\section{AIC Based \\ AMSE Based}

Estimation information

\begin{tabular}{lll}
\hline AIC & 6147.985 & 6177.137 \\
SSR & 1022170. & 1077010. \\
RMSE & $\mathbf{4 8 . 0 3 5 2 1}$ & 49.30693 \\
AMSE & 3448.010 & 3326.265 \\
\hline
\end{tabular}

Table 8

Comparison of HWMS and ETS Methods in Case of Daily Data Traffic.

\begin{tabular}{|c|c|c|c|}
\hline \multicolumn{2}{|c|}{ Exponential Smoothing Methods } & $\begin{array}{l}\text { Simple } \\
\text { HWMS }\end{array}$ & $\begin{array}{l}\text { ETS } \\
\{\mathrm{A}, \mathrm{N}, \mathrm{M}\}\end{array}$ \\
\hline \multirow{4}{*}{ Parameters } & Alpha & 0.6700 & \multirow[t]{2}{*}{0.669730} \\
\hline & Beta & 0.0000 & \\
\hline & Gamma & 0.0000 & 0.000000 \\
\hline & \multicolumn{3}{|l|}{ Phi } \\
\hline \multicolumn{3}{|c|}{ Akaike Information Criterion } & 6147.985 \\
\hline \multicolumn{2}{|c|}{ Sum of Squared Residuals } & 1021982. & 1022170 \\
\hline \multicolumn{2}{|c|}{ Root Mean Squared Error } & 48.03080 & 48.03521 \\
\hline \multicolumn{3}{|c|}{ Average Mean Squared Error } & 3448.010 \\
\hline
\end{tabular}

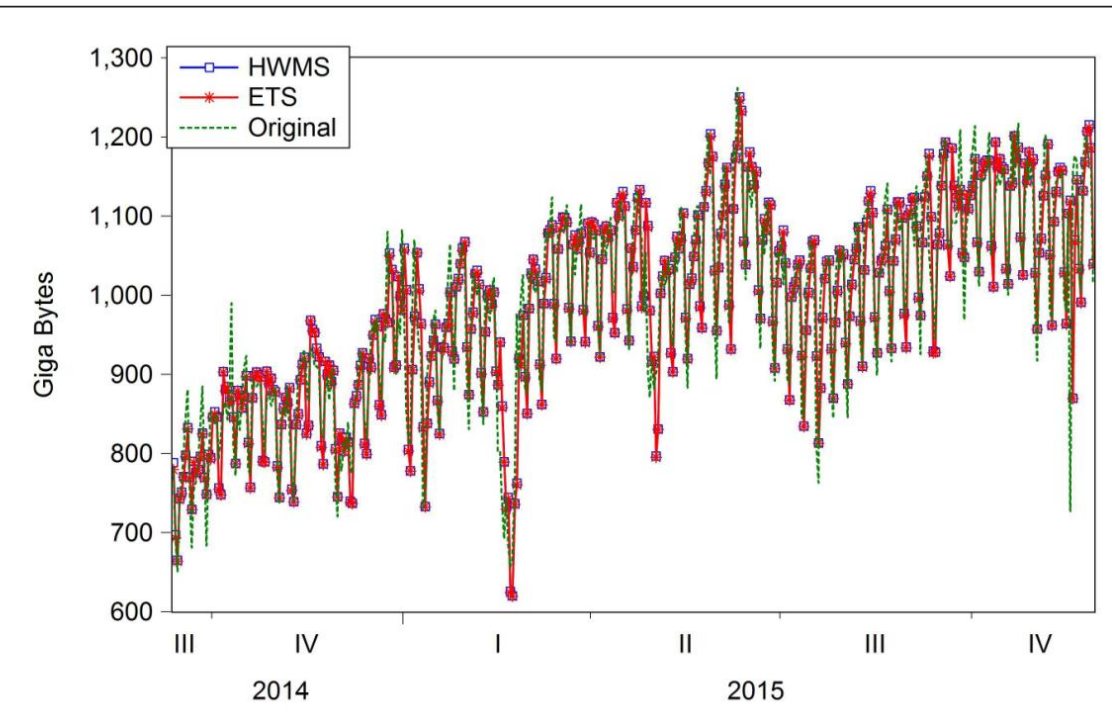

Figure 7. In-sample forecast by HWMS and ETS in case of daily data traffic. 

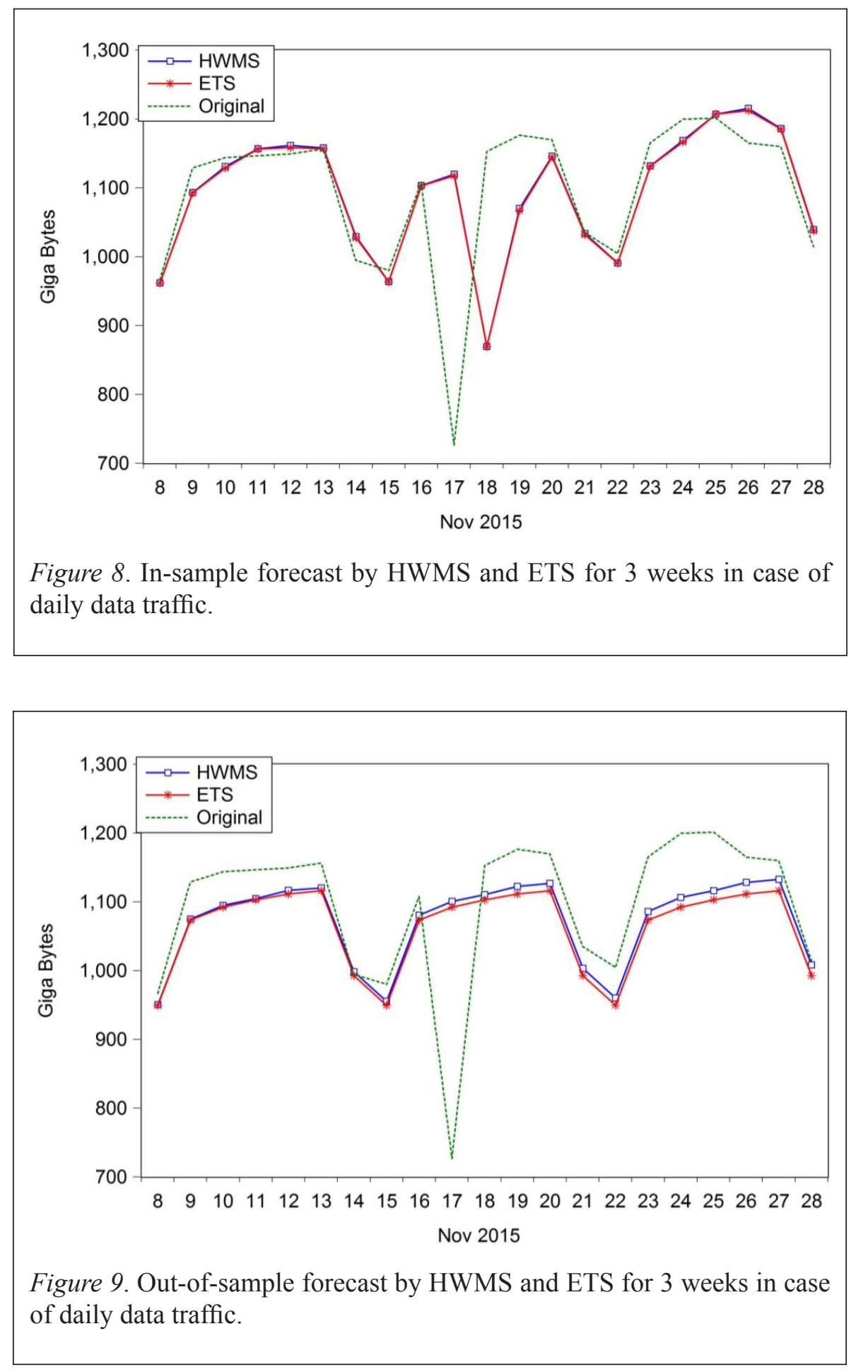


\section{CONCLUSION}

In network planning and management, short-term prediction and real-time decisions are best served by simple and low-cost prediction methods with a high accuracy. Therefore, exponential smoothing methods, such as HWMS, were applied to forecast GSM/GPRS network Erlang traffic. However, a more comprehensive study was required to evaluate the usage of various exponential smoothing methods in more types of wireless traffic, such as data, which is becoming more important along with the development of communication technology. This research applied different exponential smoothing methods that were categorized as a simple exponential smoothing method and ETS framework, to forecast cellular network traffic that included voice and data. The experiments on hourly and daily traffic collected from commercial cellular networks showed that HWMS was the best fit for cellular network traffic among other simple exponential smoothing methods that included single, double, Holt-Winters No Seasonal, and Holt-Winters Additive Seasonal. It was unsuitable for the ETS framework to forecast hourly voice traffic. However, in the case of daily voice and data traffic, the ETS found that the $\{A, N, M\}$ models provided nearly the same results as the HWMS. The HWMS forecast series was close to the original series in both hourly and daily voice traffic cases and showed a good ability to forecast daily data traffic as well. Due to the low complexity and low cost, HWMS can be applied effectively to cellular network traffic prediction. Moreover, the evaluation of HWMS can be further implemented based on the specific requirements of cellular networks.

\section{ACKNOWLEDGMENT}

This study was supported by National Natural Science Foundation of China (NSFC No.61271245).

\section{REFERENCES}

5G Vision. (2015). Retrieved from https://5g-ppp.eu/roadmaps

Fletcher, R. (1987). Practical methods of optimization (2nd ed.). New York: John Wiley \& Sons.

Hyndman, R. J., Koehler, A. B., Snyder, R. D., \& Grose, S. (2002). A state space framework for automatic forecasting using Exponential smoothing methods. International Journal of Forecasting, 18(3), 439-454. doi: 10.1016/S0169-2070(01)00110-8

Margaret, V., \& Jose, J. (2015). Exponential smoothing models for prediction of solar irradiance. International Journal of Advanced Research in 
Electrical, Electronics and Instrumentation Engineering, 4 (2), 11331139. doi: 10.15662/ijareeie.2015.0402083

Matta, P., Pant, B., \& Arora, M. (2017). All you want to know about Internet of Things (IoT). International Conference on Computing, Communication and Automation (ICCCA2017). Greater Noida, India: IEEE. doi: 10.11.09/CCAA.2017.8229999

NGMN 5G White Paper. (2015). Retrieved from https://www.ngmn.org/5gwhite-paper $/ 5 \mathrm{~g}$-white-paper.html

Qiang, L., Li, J., \& Altman, E. (2017). A novel distributed network selection scheme for heterogeneous wireless network environments. IEEE Transactions on Control of Network Systems, 4(3), 575-586. doi: 10.1109/TCNS.2016.2541338

Rahman, M. H., Salma, U., Hossain, M. M., \& Khan, M. T. F. (2016). Revenue forecasting using holt-winters exponential smoothing. Research \& Reviews: Journal of Statistics, 5(3), 19-25.

Siregar, B., Butar-Butar, I. A., Rahmat,. R.F., Andayani, U., \& Fahmi, F. (2017). Comparison of Exponential Smoothing methods in forecasting palm oil real production. Journal of Physics: Conference Series,801(1), 1-9. doi:10.1088/1742-6596/801/1/012004

Tikunov, D., \& Nishimura, T. (2007). Traffic prediction for mobile network using holt-winter's exponential smoothing. 15th International Conference on Software, Telecommunications and Computer Networks, SoftCOM 2007. Split-Dubrovnik, Croatia: IEEE. doi: 10.1109/ SOFTCOM.2007.4446113

Tran, Q. T., Ma, Z., Li, H., Hao, L., \& Trinh, Q. K. (2015). A multiplicative seasonal ARIMA/GARCH model in EVN traffic prediction. International Journal of Communications, Network and System Sciences, 8(4), 43-49. doi: 10.4236/ijens.2015.84005

Tyagi, V., \& Kumar, A. (2017). Internet of things and social networks: A survey. International Conference on Computing, Communication and Automation. Greater Noida, India: IEEE. doi: 10.1109/ CCAA.2017.8230013

Usaratniwart, E., Sirisukprasert, S., Hatti, N., \& Hagiwara, M. (2017). A case study in micro grid using adaptive enhanced linear exponential smoothing technique. 8th International Conference of Information and Communication Technology for Embedded Systems. Chonburi, Thailand: IEEE. doi: 10.1109/ICTEmSys.2017.7958776

Valakevicius, E., \& Brazenas, M. (2015). Application of the seasonal HoltWinters model to study exchange rate volatility. Inzenerine EkonomikaEngineering Economics, 26(4), 384-390.doi: 10.5755/j01.ee.26.4.5210

Yusuf-Asaju, A.W., Dahalin, Z.M., \& Ta'a, A (2018) Framework for modelling mobile networks quality of experience through big data analytics approach. Journal of ICT, 17(1) 79-113. 